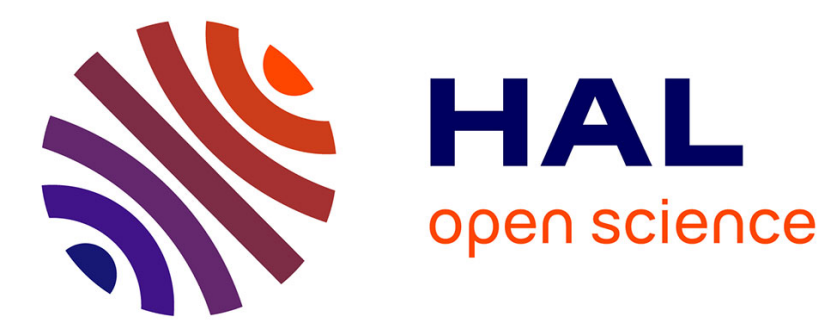

\title{
All-optical interconnects using Airy beams
}

Noémi Wiersma, Nicolas Marsal, Marc Sciamanna, Delphine Wolfersberger

\section{To cite this version:}

Noémi Wiersma, Nicolas Marsal, Marc Sciamanna, Delphine Wolfersberger. All-optical interconnects using Airy beams. Optics Letters, 2014, 39 (20), pp.5997-6000. 10.1364/OL.39.005997 . hal01082666

\section{HAL Id: hal-01082666 \\ https://hal-centralesupelec.archives-ouvertes.fr/hal-01082666}

Submitted on 4 Jun 2021

HAL is a multi-disciplinary open access archive for the deposit and dissemination of scientific research documents, whether they are published or not. The documents may come from teaching and research institutions in France or abroad, or from public or private research centers.
L'archive ouverte pluridisciplinaire $\mathbf{H A L}$, est destinée au dépôt et à la diffusion de documents scientifiques de niveau recherche, publiés ou non, émanant des établissements d'enseignement et de recherche français ou étrangers, des laboratoires publics ou privés.

\section{(ㄷ)(1)}

Distributed under a Creative Commons Attribution| 4.0 International License 


\title{
All-optical interconnects using Airy beams
}

\author{
Noémi Wiersma, * Nicolas Marsal, Marc Sciamanna, and Delphine Wolfersberger \\ Supélec, OPTEL Research Group, LMOPS (EA 4423), 2 rue Édouard Belin, 57070 Metz, France \\ ${ }^{*}$ Corresponding author: noemi.wiersma@supelec.fr
}

\begin{abstract}
We analyze numerically optical waveguide structures in photorefractive media induced by one or two incoherent counter-propagating (CP) Airy beams. Under nonlinear focusing conditions, we show that for a single Airy beam or for two CP beams with various input positions, multiple waveguiding structures can be photo-induced in the medium. Optical Gaussian beams can therefore be guided with a deflecting trajectory and/or even split into several output beams. These results enable new configurations for all-optical interconnections.
\end{abstract}

The Airy wave function has been initially suggested by Berry and Balazs [1] as a solution for the Schrödinger equation with a non-spreading wave propagation along a parabolic trajectory. First experimentally observed in free space in 2007 [2] by means of a spatial light modulator (SLM), Airy beams have been also generated using photonic crystals [3]. Later, a main interest has been to control their trajectories $[\underline{4}, 5]$ and to maintain their Airy shape [6] in nonlinear media. Their unique properties make Airy beams good candidates for micro manipulation of particles [7], imaging in scattering media [8], and waveguiding for optical routing communications [9].

Recently, the self trapping character of Airy beams in biased nonlinear media has suggested interesting dynamics such as soliton-like behaviors and interactions of co-propagating Airy functions $[10,11]$. By contrast to the co-propagating case, a counter-propagating (CP) configuration combined with the large transverse dimension of the Airy shape and their curved trajectory allow several interaction schemes where one or several lobes of the CP beams can, for example, face each other. The interaction of CP Airy beams opens therefore new interesting fields for optical interconnections.

In this Letter, we analyze numerically optical waveguide structures created in photorefractive (PR) media by one or two incoherent CP Airy beams under nonlinear self-focusing conditions. The scheme of incoherent CP beams allows strong interactions because of crosscoupling of the beams via the combined refractive index structure that is not present in the co-propagating case [12]. When a positive external electrical field is applied on the PR crystal, we show that for a single Airy beam or for two CP beams even strongly misaligned, multiple waveguiding structures are photo-induced. In that way, an optical Gaussian beam can be linearly guided along a deflecting trajectory or split into several output beams.

Our typical interaction scheme is depicted on Fig. 1 . It illustrates the linear propagation of two 1D CP Airy beams in an unbiased PR crystal. Airy profiles, $F(x, z)$ and $B(x, z)$, are given by the following equations:

$$
F(x, z=0)=F_{0} A i\left(\frac{x}{x_{0}}+d\right) \exp \left(a\left(\frac{x}{x_{0}}+d\right)\right)
$$

$$
B(x, z=L)=B_{0} A i\left(\frac{x}{x_{0}}\right) \exp \left(a \frac{x}{x_{0}}\right),
$$

where $F_{0}$ and $B_{0}$ correspond to the wave amplitudes of respectively the forward beam $F$ and the backward beam $B, A i$ represents the Airy function, $x_{0}$ an arbitrary transverse scale, $a$ the truncation factor, and $d$ the additional normalized shifting distance between the initial input beams $F$ and $B$. CP beams are herein defined by their opposite propagation directions along the longitudinal $z$ axis [Fig. 1]. Each Airy beam is formed of successive lobes, the first order being the main lobe. The nonlinear propagation of these two incoherent $\mathrm{CP}$ beams can be expressed as follows [13]:

$$
\begin{gathered}
i \partial_{z} F+\partial_{x}^{2} F=\Gamma E_{0} F, \\
-i \partial_{z} B+\partial_{x}^{2} B=\Gamma E_{0} B,
\end{gathered}
$$

where $\Gamma=\left(k n_{0} x_{0}\right)^{2} r_{\text {eff }} E_{e}$ is the nonlinear photorefractive coupling strength $\left(r_{\text {eff }}\right.$ is the effective component of the electro-optic tensor and $E_{e}$ the external electric field), and $E_{0}$ is the homogeneous part of the $x$-component of the photorefractive space-charge field. The temporal evolution of $E_{0}$ is calculated using a relaxation-type dynamics given by $\tau \partial_{t} E_{0}+E_{0}=-I_{0} /\left(1+I_{0}\right)$, where $\tau$ is the relaxation time of the crystal and $I_{0}=|F|^{2}+|B|^{2}$. A positive external electrical field is applied along the $c$ axis of the crystal (parallel to the $x$ axis [Fig. 1]). This type of interaction enables us to optically induce complex

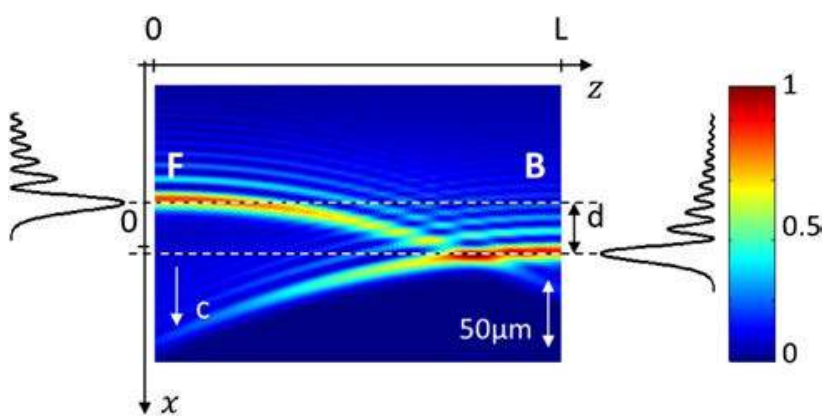

Fig. 1. Typical interaction scheme of two CP Airy beams in an unbiased photorefractive crystal. 


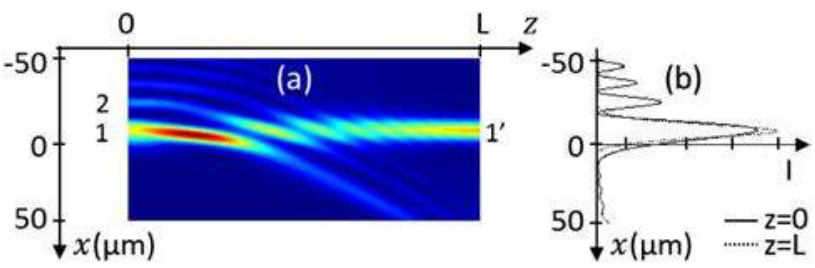

Fig. 2. (a) Normalized intensity distribution of a self-focused Airy beam propagating in a PR crystal at time $t_{f}=6 \tau$ and (b) corresponding transverse intensity profiles.

waveguiding structures in the PR nonlinear material through the Pockels effect [14]. The induced refractive index distribution is then related to the combination of the multiplexed focused Airy beams $F$ and $B$.

The induced refractive index profile is numerically simulated via a fast Fourier transform beam propagation method. We fix the crystal length $L=1 \mathrm{~cm}, \Gamma=9$, $a=0.09, x_{0}=7.5 \mu \mathrm{m}$, the normalized input energy of a beam $F_{0}=\sqrt{2.5}$, and keep $B_{0}$ and $d$ as free parameters.

We first consider optical waveguides created by a single Airy beam $F\left(B_{0}=0\right)$ propagating from $z=0$ to $z=$ $L(z=0 \rightarrow L)$ with a focusing bias electric field. Figure 2 displays the intensity of the self-focused Airy beam. The results concern the stationary state that is reached after a transient duration equal to $6 \tau$. At $z=0$, we indicate only two positions of high index variations corresponding to the two first lobe orders of the input Airy function (1,2 in Fig. 2). Interestingly and similar to the references $[\underline{10}, 15]$, the initial Airy beam with its curved trajectory turns into an "off-shooting" soliton as it propagates along the $z$ axis (1' at $z=L$ in Fig. 2).

This simple configuration created by a single Airy beam under high-focusing conditions raises the question whether the photo-induced structure can be used for various linear guidings of Gaussian beams. To test the waveguide structure in Fig. 2, we now inject at the different input positions $\left(1,2,1^{\prime}\right)$ a Gaussian probe beam with its size matching with the photo-induced waveguide at each input position $\left(1,2,1^{\prime}\right)$. We consider the linear propagation by simulating Eq. (3) or Eq. (4) and keeping the space-charge field $E_{0}$ equal to the stationary $E_{0}(x, z)$ resulting from the nonlinear propagation of the Airy beam. The crystal length $L=1 \mathrm{~cm}$ corresponds to $3.3 L_{d}$, with $L_{d}=2 k x_{0}^{2}$ the characteristic diffraction length of the Gaussian beam $\left(n_{0}=2.3, \lambda=532 \mathrm{~nm}\right)$. The results show two effective waveguides with different output structures: $1 \rightarrow 1^{\prime}$ and $1^{\prime} \rightarrow(1,2)$ [Figs. 3(a1)-3(b1), 3(a2)-3(b2)]. The first one [Figs. 3(a1)-3(b1)] guides $66 \%$ of the initial energy along the crystal [red line, Fig. 3(b1)]. Based on Fig. 2(a), we stress that the waveguide $1 \rightarrow 1^{\prime}$ is first formed by the self-focused main Airy lobe, then by the "off-shooting" soliton which no longer satisfies the Airy beam's properties but rather the ones of a self-focused Gaussian beam [15]. This guiding differs from the situation analyzed in [9] in that, under high-focusing conditions, the output position of the waveguide $1 \rightarrow 1^{\prime}$ does not relate to the deflection of the Airy beam but is rather determined by the position of the "off-shooting" soliton. The scenario is different when the Gaussian probe beam is injected in $1^{\prime}$ and propagates in the other direction $(z=L \rightarrow 0)$ [Figs. 3(a2)-3(b2)]. We show that the energy
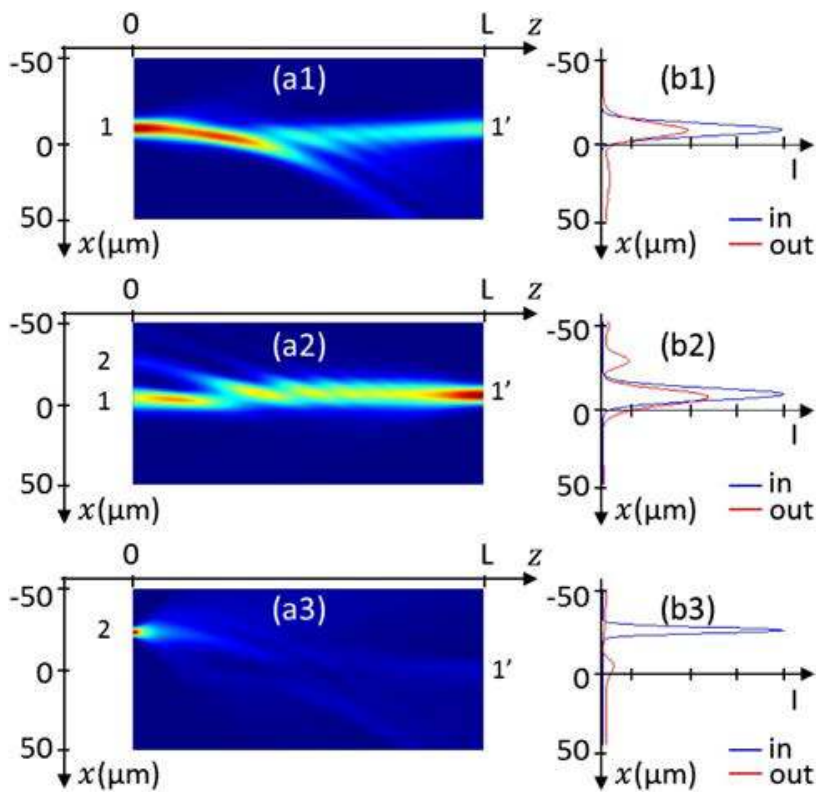

Fig. 3. Linear probe beam propagation in the waveguide structure of Fig. 2: (a1) intensity distribution and (b1) transverse profiles of Gaussian beam guided along $1 \rightarrow 1^{\prime}$, (a2)-(b2) guided along $1^{\prime} \rightarrow(1,2)$, (a3)-(b3) guided along $2 \rightarrow 1^{\prime}$.

of the initial Gaussian beam splits now into two outputs: $30 \%$ in 1 and $20 \%$ in 2 . The demultiplex structure $\left(1^{\prime} \rightarrow(1,2)\right)$ allows for a large spacing between the two outputs (1 and 2$): \approx 3 x_{0}$ [Fig. 3(b2)]. It is worth emphasizing that such a waveguide with one input and two outputs cannot be achieved with a single Gaussian beam and therefore utilizes the unique properties of the Airy beam. The waveguide $2 \rightarrow 1^{\prime}$ does not present strong guiding of the Gaussian probe beam along the crystal [Figs. 3(a3)-3(b3)]. This low guiding efficiency can be explained by the lower refractive index structure photo-induced by the second lobe order of the Airy beam at $z=0$ and by the asymmetrical waveguide structure between $z=0$ and $z=L$. Those results demonstrate that, although the waveguiding is bidirectional (i.e., a Gaussian probe beam on both crystal sides is guided through the structure), the asymmetry of the waveguide leads to different output positions and guiding efficiencies depending on the input position of the probe beam.

To enlarge the possible interconnection schemes, we now study the interactions of two self-focused CP Airy beams in the PR crystal. We restrict our investigation to two configurations: the first one where the two $\mathrm{CP}$ Airy beams ( $F$ and $B$ with $F_{0}=B_{0}$ ) are strictly aligned (transverse shift $d=0$, [Figs. 4(a1)-(b1)]), and second with a small misalignment $d=2$ [Figs. 4(a2)-4(b2)]. Similarly to Fig. 2, input positions created by the Airylobes $(B)$ are named 3 for the main lobe, 4 for the second, and $3^{\prime}$ for the "off-shooting" soliton coming from $B$.

Results for F and B initially aligned $(d=0)$ : First, it is worth noting that the backward beam modifies the trajectory of the forward beam $F$ [Figs. 4(a1)-4(b1)]. Indeed, at $z=L$, the position of the "off-shooting" soliton $1^{\prime}$ (respectively $3^{\prime}$ at $z=0$ ) is not the same compared to the situation with only one Airy beam (Fig. 2). We notice an additional drift of $2 x_{0}$. This configuration enables therefore larger transverse distances for optical guiding 


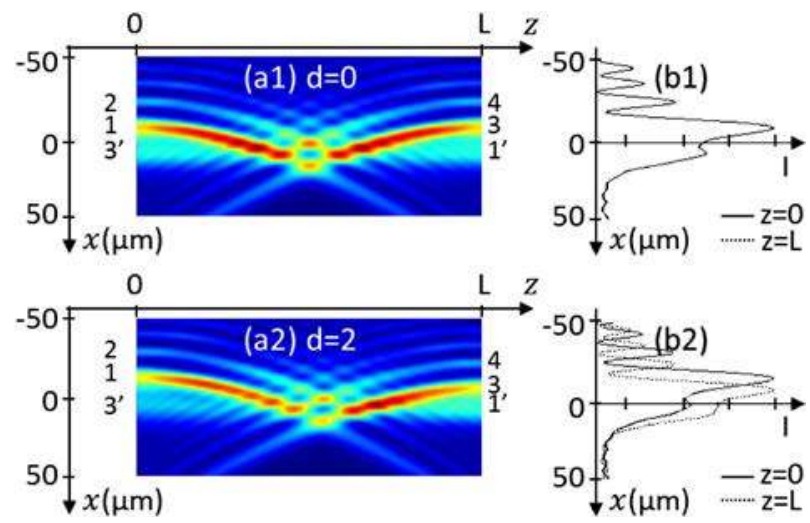

Fig. 4. (a1)-(a2) Normalized intensity because of the interaction of CP self-focused Airy beams in a PR crystal at time $t_{f}=$ $8 \tau$ and (b1)-(b2) corresponding transverse intensity profiles.

and waveguide configurations: $1 \rightarrow 1^{\prime}$ [Figs. $5(\mathrm{a} 1)-5(\mathrm{~b} 1)$ ] or $1^{\prime} \rightarrow(1,2)$ and respectively $3 \rightarrow 3^{\prime}$ or $3^{\prime} \rightarrow(3,4)$ [Figs. 5(a2)-5(b2)]. Although the interest of this CP configuration is also the symmetry of the waveguide structure, we notice that the waveguide $1 \rightarrow 1^{\prime}$ [Figs. 5(a1)-5(b1)] created by $F$ and $B$ transmits half less energy than the one created by a single $F$ [Figs. 3(a1)-3(b1)]. The strong interaction due to cross-coupling of the beams via the combined refractive index structure and energy flows between not only the main CP lobes but also the neighbors [Fig. 4(a1)], [4] can explain the possible energy losses on the depicted structures. As a main conclusion we can stress that the use of two CP Airy beams allows for achieving complex waveguiding structures that would otherwise require the $\mathrm{CP}$ interactions of more than two Gaussian beams.

Results for F and B initially misaligned $(d=2)$ : If the two input beams are initially shifted [Figs. 4(a2)-4(b2)], additional interactions between the self-focused CP Airy beams appear. The asymmetry between the initial Airy beams' positions is because of a misalignment of $2 x_{0}$ between the successive lobes of $F$ and $B$. Regarding the "off-shooting" solitons (1' and $3^{\prime}$ [Figs. 4(a2)-4(b2)]), if we consider their previous positions for $d=0$ [Figs. 4(a1)-4(b1)], the additional $d$-shift brings the position of $1^{\prime}$ closer to the main lobe of $B(3)$. By contrast, the
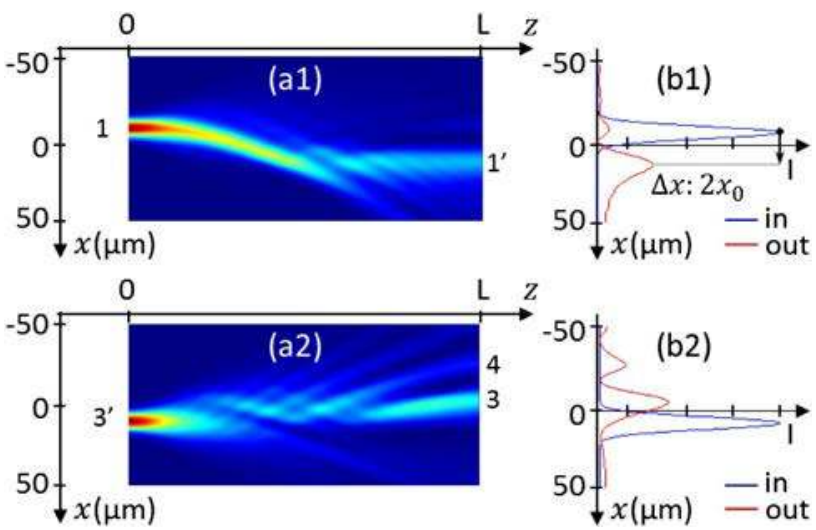

Fig. 5. Linear probe beam propagation in the waveguide structure of Figs. 4(a1)-4(b1): (a1) Intensity distribution and (b1) transverse profiles of a Gaussian beam guided along $1 \rightarrow 1^{\prime}$, (a2)-(b2) guided along $3^{\prime} \rightarrow(3,4)$.
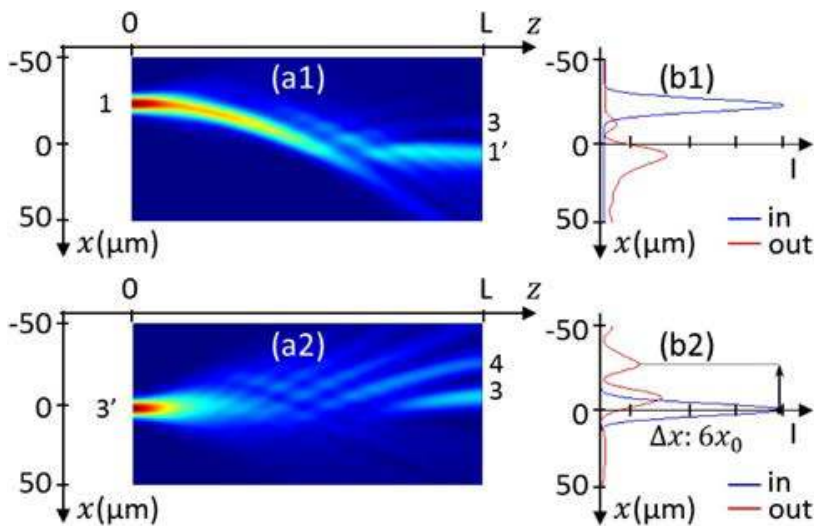

Fig. 6. Linear probe beam propagation in the waveguide structure of Figs. 4(a2)-4(b2): (a1) intensity distribution and (b1) transverse profiles of a Gaussian beam guided along $1 \rightarrow\left(1^{\prime}, 3\right)$, (a2)-(b2) guided along $3^{\prime} \rightarrow(3,4)$.

"off-shooting" soliton's position 3 ' is shifted away from 1 . In this configuration, higher energy is exchanged within the CP main and secondary lobes. If a probe beam is injected into the PR crystal, different output cases are observed [Fig. 6]. Similar to the structure generated when $d=0$, if the probe beam is injected into inputs formed by "off-shooting" solitons ( $1^{\prime}$ or $\left.3^{\prime}\right)$, it will split into two beams at the Airy lobes positions on the other side of the crystal with input-output shifts up to $6 x_{0}\left(3^{\prime} \rightarrow(3,4)\right.$ [Figs. 6(a2)-6(b2)]). However, if the Gaussian beam is first guided by a main Airy lobe (1 or 3), we not only observe, as in Figs. 5(a1)-5(b1), an output beam at the soliton position ( $1^{\prime}$ or $\left.3^{\prime}\right)$, but also a second one appears at the position of the main lobe of the CP beam (3 or 1) [Figs. 6(a1)-6(b1)]. Such a configuration where interlobe interaction areas inside the crystal are possible, enables creating 1-to-2-demultiplexers guiding up to half the energy of the input beam (outputs: 33\%-15\%) with an important transverse shift $x$. The resulting waveguide structures show interesting features. First the waveguide occurs although the positions of the two input beams are largely shifted $\left(2 x_{0}\right)$. The same simulation, but using shifted Gaussian beams at the entrances 1 and 3, does not create any waveguide. Second the interaction scheme allows for controlling the spatial positions of the waveguide outputs by varying the transverse shift $d$ between the CP input beams.

The high-focusing conditions of the propagation medium has led to a soliton-like behavior of the Airy beam, and our interaction studies have shown first coupling effects between the CP Airy beams resulting in different waveguiding possibilities. It is worth stressing that all previous cases correspond to stationary situations in that the waveguide remains fixed when time increases above a transient duration of the order of the relaxation time of the crystal [Fig. 7(a)]. Although not discussed in detail, we do find parameters where the waveguiding is not stationary anymore. In Figs. $\underline{7(b)}$ and $\underline{7(\mathrm{c})}$, when

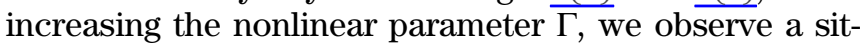
uation where the intensity profile at the crystal output varies periodically [Fig. $\underline{7(b)}$ ] or irregularly [Fig. $\underline{7(\mathrm{c})}]$ over time.

To conclude, we have demonstrated waveguiding achieved by the nonlinear interactions of CP Airy beams. 


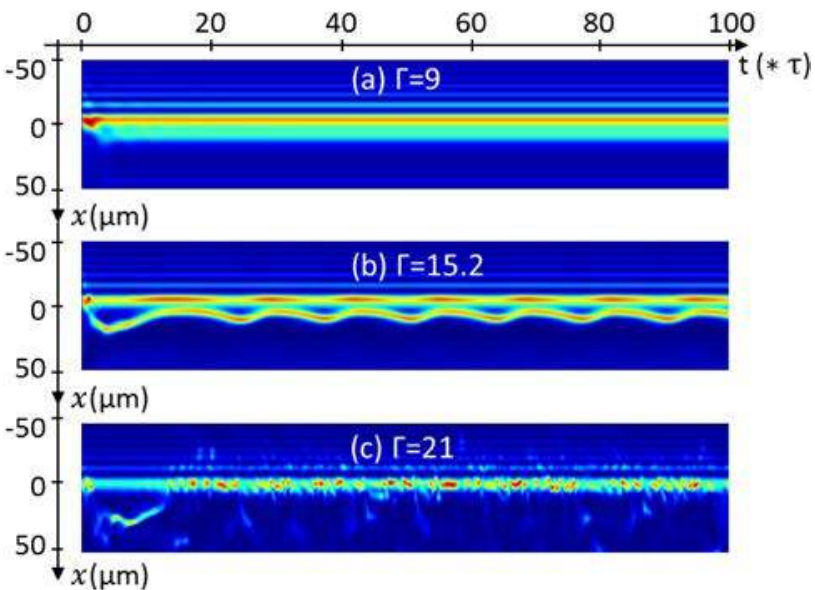

Fig. 7. Spatiotemporal dynamics for CP aligned Airy beams. Normalized transverse intensity at $z=L$ versus time: (a) stationary $(\Gamma=9)$, (b) time periodic $(\Gamma=15.2)$, and (c) irregular $(\Gamma=21)$.

In comparison with waveguide structures created by Gaussian beams with the same dimensions and focusing conditions [12], the interaction schemes between two Airy beams offer larger waveguide possibilities thanks to their unique Airy shape and properties: (1) A single Airy beam photo-induces a waveguide with two outputs; (2) two CP Airy beams induce multiple output waveguiding structures that cannot be achieved with only two CP Gaussian beams; and (3) the resulting waveguiding remains possible even for transverse shifts of the interacting beams that by far exceed the beam waist. The numerical simulations presented in this Letter motivate an experimental implementation of CP Airy beams induced optical routing. In addition the first spatiotemporal dynamics observed in a CP Airy beam system encourage further investigation into the relations between the Airy properties and the solitonic interactions.

The authors acknowledge the support of Conseil Régional de Lorraine and IAP P7/35 (BELSPO).

\section{References}

1. M. Berry and N. Balazs, Am. J. Phys. 47, 264 (1979).

2. G. A. Siviloglou and D. N. Christodoulides, Opt. Lett. 32, 979 (2007).

3. T. Ellenbogen, N. Voloch-Bloch, A. Ganany-Padowicz, and A. Arie, Nat. Photonics 3, 395 (2009).

4. Y. Hu, P. Zhang, C. Lou, S. Huang, J. Xu, and Z. Chen, Opt. Lett. 35, 2260 (2010).

5. I. Dolev, T. Ellenbogen, and A. Arie, Opt. Lett. 35, 1581 (2010).

6. S. Jia, J. Lee, J. W. Fleischer, G. A. Siviloglou, and D. N. Christodoulides, Phys. Rev. Lett. 104, 253904 (2010).

7. J. Baumgartl, T. Cizmar, M. Mazilu, V. C. Chan, A. E. Carruthers, B. A. Capron, W. McNeely, E. M. Wright, and K. Dholakia, Opt. Express 18, 17130 (2010).

8. F. O. Fahrbach, P. Simon, and A. Rohrbach, Nat. Photonics 4, 780 (2010)

9. P. Rose, F. Diebel, M. Boguslawski, and C. Denz, Appl. Phys. Lett. 102, 101101 (2013).

10. I. Kaminer, M. Segev, and D. N. Christodoulides, Phys. Rev. Lett. 106, 213903 (2011).

11. Y. Zhang, M. Belić, Z. Wu, H. Zheng, K. Lu, Y. Li, and Y. Zhang, Opt. Lett. 38, 4585 (2013).

12. M. Petrović, M. Belić, C. Denz, and Y. S. Kivshar, Laser Photon. Rev. 5, 214 (2011).

13. M. Belić, P. Jander, K. Motzek, A. Desyatnikov, D. Jović, A. Strinić, M. Petrović, C. Denz, and F. Kaiser, J. Opt. B 6, S190 (2004).

14. M. Alonzo, C. Dan, D. Wolfersberger, and E. Fazio, Appl. Phys. Lett. 96, 121111 (2010).

15. Y. Hu, Z. Sun, D. Bongiovanni, D. Song, C. Lou, J. Xu, Z. Chen, and R. Morandotti, Opt. Lett. 37, 3201 (2012). 